\title{
EFEITO DO GRADIENTE DE SALINIDADE NA TAXa FOTOSSINTÉTICA DE POLYSIPHONIA SUBTILISSIMA, CLADOPHORA VAGABUNDA E ULVA FLEXUOSA SUbSP. Flexuosa na Lagoa Rodrigo de Freitas, Rio de Janeiro, BrasiL ${ }^{1}$
}

\author{
Rafael R. Loureiro ${ }^{2} \&$ Renata P. Reis, 3
}

\begin{abstract}
Resumo
(Efeito do gradiente de salinidade na taxa fotossintética de Polysiphonia subtilissima, Cladophora vagabunda e Ulva flexuosa subsp. flexuosa na Lagoa Rodrigo de Freitas, Rio de Janeiro, Brasil) Este estudo tem o intuito de testar o desempenho fotossintético das espécies de algas bentônicas mais representativas da Lagoa Rodrigo de Freitas (Polysiphonia subtilissima, Cladophora vagabunda e Ulva flexuosa subsp. flexuosa) em diferentes salinidades, a fim de verificar seus limites de tolerância. Cada amostra foi incubada nas salinidades 10, 15 e 25. Para cada salinidade foram realizadas quatro repetições (luz e escuro). Amostras de $10 \mathrm{mg}$ foram utilizadas para cada espécie, durante aproximadamente 30 minutos de incubação, sob irradiância de $500 \pm$ $5 \mathrm{mmol} \cdot \mathrm{m}^{-2} \cdot \mathrm{s}^{-1}$. Polysiphonia subtilissima e Cladophora vagabunda tiveram maior produção fotossintética na salinidade de $15(P<0,05)$. Ulva flexuosa subsp. flexuosa não apresentou diferença em sua taxa fotossintética nas três salinidades $(P=0,09)$. Os resultados obtidos sugerem que alterações futuras na salinidade da lagoa poderão causar modificações na dinâmica das populações das algas locais.
\end{abstract}

Palavras-chave: lagoas costeiras, dinâmica de população, fotossíntese.

\section{Abstract}

(Effect of salinity gradient on photosynthetic rate of Polysiphonia subtilissima; Cladophora vagabunda and Ulva flexuosa subsp. flexuosa at Rodrigo de Freitas Lagoon, Rio de Janeiro, Brazil) This study aimed to test the photossynthetic performance of the most representative benthonic macroalgae species at the Rodrigo de Freitas Lagoon (Polysiphonia subtilissima, Cladophora vagabunda and Ulva flexuosa subsp. flexuosa) in response to different salinities in order to verify their tolerance limits. Each sample was tested under salinities 10, 15 and 25. Four replicates (light and dark) were used for each salinity. Samples of $10 \mathrm{mg}$ were used for each species, during 30 minutes incubation, under irradiance of $500 \pm 5 \mathrm{mmol} \cdot \mathrm{m}^{-2} \cdot \mathrm{s}^{-1}$. P. subtilissima and C. vagabunda presented higher photosynthetic production in the salinity of $15(P<0.05)$. U. flexuosa subsp. flexuosa did not differ in its photosynthetic rate under the three salinities sampled $(P=0.09)$. The present results suggest that future alterations related to the lagoon's salinity may cause alterations in the populations dynamics of the local algae. Key words: coastal lagoons, population dynamics, photosynthesis.

\section{INTRODUÇÃO}

A disponibilidade de luz, nitrogênio e a condição limitante que a salinidade impõe são considerados os principais fatores que controlam a produtividade de macroalgas em ambientes estuarinos (McGlathery \& Pedersen 1999). Tais fatores variam consideravelmente em ambientes naturais, principalmente devido à influência antrópica (McGlathery et al. 1996, 1997). Em ambientes estuarinos, a disponibilidade de nutrientes pode variar muito em um curto espaço de tempo (de horas a dias) como em ciclos sazonais, assim como os fatores que limitam o crescimento, como salinidade, em períodos de extrema seca ou chuva excessiva (Ramus \& Venable 1987). Macroalgas que se encontram neste tipo de ambiente devem apresentar mecanismos que contrabalançam a disponibilidade de recursos e os fatores limitantes com o seu crescimento e a sua produtividade.

A distribuição de algas oportunistas como as do gênero Ulva e Cladophora, comuns em zonas estuarinas (Poole \& Raven 1997), assim como da Rodophyta Polisyphonia subtilissima Mont. podem ser afetadas pela variabilidade da salinidade causadas pela maior contribuição de água marinha ou doce, como observadoem estudos desenvolvidos, tanto em laboratórios quanto em campo, que

Artigo recebido em 06/2007. Aceito para publicação em 02/2008.

${ }^{1}$ Parte da Dissertação de Mestrado do primeiro autor desenvolvida no Curso de Pós-Graduação em Botânica do Instituto de Pesquisas Jardim Botânico do Rio de Janeiro.

${ }^{2}$ Instituto de Pesquisas Jardim Botânico do Rio de Janeiro. R. Pacheco Leão 915, Rio de Janeiro, RJ, 22460-030, Brasil.

${ }^{3}$ Autor para correspondência: rreis@jbrj.gov.br 
apresentaram salinidade ótima para cada espécie destes gêneros de 18-22 (Martins et al. 1999; Ulva intestinalis [=Enteromorpha intestinalis] spp.), 24 (Kim \& Lee 1996; Ulva intestinalis [=E. intestinalis]), e 27 (Taylor et al. 2001; Ulva linza $[=E$. linzaI]), 18 (Wiencke \& Davenport 1987; Wiencke et al. 1992; C. rupestris), 15 (Reed et al. 1980; Reed 1983; 1984; P. lanosa). Estes valores representam as condições ideais de salinidade determinando elevada produtividade (fotossíntese) e conversão em biomassa.

Uma vez que o governo do estado do Rio de Janeiro pretende alargar a entrada do Canal do Jardim de Alah, que é a ligação da laguna com o mar, conseqüentemente a laguna receberá um maior aporte de água marinha (COPPE 2006), que certamente modificará a dinâmica do ambiente. Na Lagoa Rodrigo de Feitas, as espécies de macroalgas dominantes, são Polysiphonia subtilissima, Cladophora vagabunda e Ulva flexuosa subsp. flexuosa (Loureiro 2007). Este estudo tem o intuito de testar o desempenho fotossintético destas espécies em diferentes salinidades a fim de verificar seus limites de tolerância.

\section{Material e Métodos}

A Lagoa Rodrigo de Freitas (22 $57^{\prime}$ '02" a 22 58'09'"S e 4311'09'-4313'03'W) apresenta um espelho de água de $2,2 \mathrm{~km}^{2}$ e volume de aproximadamente 6 milhões de $\mathrm{m}^{3}$ (FEEMA 2002). É caracterizada como uma laguna abrigada, com fundo lodoso, ou como uma bacia de acumulação de águas pluviais, de aspecto estuarino com salinidade média de 15 . A água do mar penetra na laguna no período da preamar das marés de sizígia, pelo Canal do Jardim de Alah que é a única ligação com o mar. Os rios que deságuam na Lagoa Rodrigo de Freitas são: o Rio dos Macacos, com área de drenagem de 7,2 km², o Rio Rainha, com 4,3 km² e o Rio Cabeças, que tem a menor área de drenagem, com 1,9 km² (FEEMA 2002). Ruppia maritima L. ocorre fixa no fundo lodoso da laguna (Oliveira et al. 1983).

Considerou-se a nomenclatura taxonômica adotada por Hayden et al. (2003), que devido evidencia molecular reuniu os gêneros Ulva e
Enteromorpha como originalmente proposto por Linneaus.

Foram analisadas amostras de Ulva flexuosa (Wulfen) J. Agardh subsp. flexuosa, Cladophora vagabunda (L.) van den Hoek e Polisyphonia subtilissima Mont. Os exemplares utilizados no experimento foram coletados aleatoriamente em locais próximos as margens da laguna. No local, cada amostra foi limpa de epífitas e de organismos epibiontes e disposta em frascos de DBO, com $300 \mathrm{ml}$ de capacidade de água nas seguintes salinidades: 10, 15 e 25. Foi utilizada a Escala Prática de Salinidade (PSS = Practical Salinity Scale), mensurada com o uso de refratômetro manual, marca Atago, modelo S10. Salinidade 10 representa o extremo de contribuição de água doce, 15 a média atual da salinidade da laguna e 25 a média da salinidade que provavelmente poderá ocorrer, caso o canal de ligação entre a laguna e o mar seja alargado (COPPE 2006). As salinidades foram obtidas pelo método de congelamento e descongelamento da água do mar (Oliveira et al. 1995), no qual as primeiras alíquotas descongeladas são as mais salinas.

Para cada salinidade foram realizadas quatro repetições de luz e quatro repetições de escuro no mês de setembro de 2006. Aágua utilizada foi filtrada em filtro de éster celulose com 0,45 mm de poro da marca Millipore. Posteriormente, cada litro foi esterilizado em forno microondas por 10 minutos, na potência máxima. Aágua foi mantida em quarto escuro para a redução do oxigênio dissolvido. Amostras de $10 \mathrm{mg}$ de cada espécie foram utilizadas, as quais permaneceram, em regra, por 30 minutos em incubação, até a visualização de pequenas bolhas na superfície das ramificações, sob irradiância de $500 \pm 5 \mu \mathrm{mol}$ de fótons. $\mathrm{m}^{-2} \cdot \mathrm{s}^{-1}$. Esta é a intensidade que reproduz a profundidade onde essas algas são encontradas ao longo da laguna $(50 \mathrm{~cm}$ de profundidade em relação à linha de água). A irradiância foi obtida com medidor de irradiância modelo Datalogger Licor 1000. O sensor do aparelho foi colocado abaixo das malhas. O oxigênio dissolvido foi medido através de oxímetro portátil (medição de mg.1) modelo Q-408P Quimis. 
Os valores de fotossíntese brutos, líquidos e respiração foram calculados através das seguintes fórmulas sugeridas em Czaplewski, \& Parker (1974): Fotossíntese Bruta (garrafa iluminada - garrafa escura)/(tempo em horas $x$ coeficiente de fotossíntese). O coeficiente de fotossíntese apresenta um valor fixo de 1,2; Fotossíntese Líquida (garrafa iluminada - garrafa inicial)/(tempo em horas $\mathrm{x}$ coeficiente de fotossíntese); Respiração (garrafa inicial - garrafa escura) $\mathrm{x}$ coeficiente de respiração/tempo em horas. O coeficiente de respiração apresenta um valor fixo de 1 . Os valores de respiração e as taxas foram medidas por oxigênio e convertidas para carbono. As taxas explicitadas no texto referem-se à fotossíntese líquida.

Para o tratamento dos dados de produtividade de cada espécie foi testada a normalidade (teste de Shapiro-Wilk) e a homogeneidade das variâncias (Teste de Cochran) para utilizar a análise de variância unifatorial (ANOVA) que verificou a diferença da produtividade nas salinidades testadas. Os resultados estão representados graficamente em média, erro padrão e desvio padrão. O intervalo de confiança para todos os testes de significância foi de $95 \%(P=0,05)$. Foram utilizados os programas Office-Excel 2002 Microsoft ${ }^{\circledR}$ e Statistica 6.0 StatSoft $\AA$.

\section{Resultados}

Ulva flexuosa subsp. flexuosa, Cladophora vagabunda e Polysiphonia subtilissima apresentaram diferentes respostas nas salinidades testadas. Quando as taxas fotossintéticas obtidas nas três salinidades foram comparadas, $P$. subtilissima (ANOVA, $F=5,26$, $P<0,05$, n = 4, Fig. 1) e $C$. vagabunda (ANOVA, $F=7,23, P<0,05$, n = 4, Fig. 2) apresentaram maior produção fotossintética na salinidade de 15 , enquanto nas demais salinidades ( 10 e 25 ) elas não diferiram $(C$. vagabunda ANOVA, $F=$ $0,32, P=0,14, \mathrm{n}=4$ e $P$. subtilissima ANOVA, $F=0,55, P=0,19, \mathrm{n}=4)$. Diferentemente, $U$. flexuosa subsp. flexuosa não apresentou diferença em suas taxas fotossintéticas (ANOVA, $\mathrm{F}=0,67, P=0,09, \mathrm{n}=4$, Fig. 3).

A fotossíntese líquida de $P$. subtilissima $\left(16,6 \pm 0,59 \mathrm{mgCm}^{-3} \mathrm{~h}^{-1}\right)$ na salinidade 15 , foi maior do que a de $C$. vagabunda $(13,3 \pm 0,61$ $\mathrm{mgCm}^{-3} \mathrm{~h}^{-1}$ ) e de $U$. flexuosa subsp. flexuosa $\left(12,6 \mathrm{mgCm}^{-3} \mathrm{~h}^{-1}\right.$, ANOVA, $F=3,47, P<0,05$, $\mathrm{n}=4$, Fig. 3). Por outro lado, as produtividades de C. vagabunda e de U. flexuosa não diferiram tanto na salinidade 10 (ANOVA, $F$ $=0,09, P=0,06, \mathrm{n}=4$ ), quanto na 15 (ANOVA, $F=0,43, P=0,07, \mathrm{n}=4$ ) e na 25 (ANOVA, $F$ $=0,27, P=0,08, \mathrm{n}=4)$. Nas salinidades de 10 (ANOVA, $F=0,08, P=0,07, \mathrm{n}=4$ ) e 25 (ANOVA, $F=0,10, P=0,06, \mathrm{n}=4$ ), todas as espécies mostraram valores semelhantes de produtividade, ou seja, $11,02 \pm 0,45 \mathrm{mgCm}^{-3} \mathrm{~h}^{-1}$ (Figs. 1, 2 e 3).

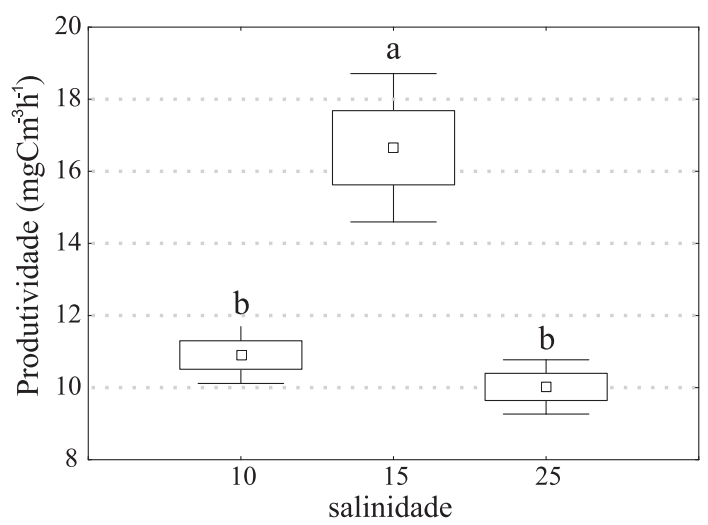

Figura 1 - Taxas fotossintéticas líquidas de Polysiphonia subtilissima em diferentes salinidades. As letras indicam as diferenças estatísticas (ANOVA, $P<0,05, \mathrm{n}=4$, Tukey) e $\square=$ média, $\square= \pm$ erro padrão e $I= \pm$ desvio padrão.

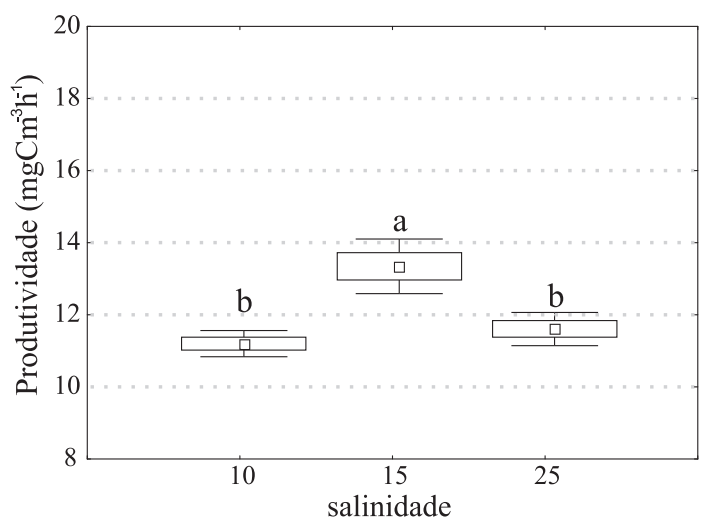

Figura 2 - Taxas fotossintéticas líquidas de Cladophora vagabunda em diferentes salinidades. As letras indicam as diferenças estatísticas (ANOVA, $P<0,05, \mathrm{n}=4$, Tukey). $\square=$ média, $\square= \pm$ erro padrão e $I= \pm$ desvio padrão. 


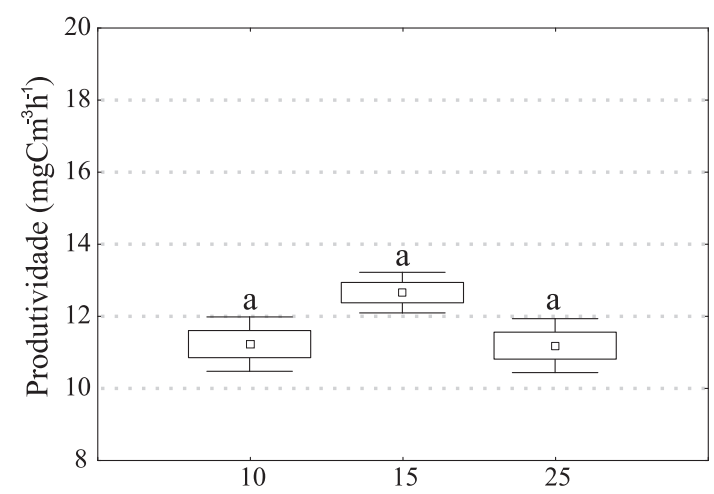

Figura 3 - Taxas fotossintéticas líquidas de Ulva flexuosa subsp. flexuosa em diferentes salinidades. As letras indicam as diferenças estatísticas (ANOVA, $P<0,05, \mathrm{n}=4$, Tukey). $\square=$ média,$\square= \pm$ erro padrão e $I= \pm$ desvio padrão.

\section{Discussão}

Atualmente na Lagoa Rodrigo de Freitas, o aporte de água do mar, principalmente em dias de ressaca, é controlado pelo fechamento das comportas do canal do Jardim de Alah e quando este fechamento coincide com os dias chuvosos, ocorre uma maior contribuição de água doce do que marinha, observada através de medições a cada dois dias nestas ocasiões (Loureiro 2007).

As respostas da produtividade de Polysiphonia subtilissima, submetida às salinidades de 10 e 25 , mostraram a efetiva influência da salinidade na performance fotossintética desta espécie na Lagoa Rodrigo de Freitas. Este resultado corrobora as afirmações de Gessner \& Schramm (1971) sobre a existência de valores ótimos de salinidade para a fotossíntese, a respiração e o crescimento para cada espécie. Nesse caso, sugere-se a ocorrência de um ecótipo de $P$. subtilissima nesta laguna, explicada pela sua maior produtividade na salinidade 15 .

A ausência de diferença significativa na produtividade de Ulva flexuosa subsp. flexuosa e de Cladophora vagabunda entre as salinidades de 10 e 25, mostra a tolerância destas espécies as variações de salinidade, confirmando a sua classificação como espécies oportunistas e de rápida recuperação quando expostas à situações estressantes (Wiencke \& Davenport 1987; Wiencke et al 1992). Nesta situação, $U$. flexuosa parece ser a espécie mais eurihalina, uma vez que não houve diferença significativa entre as três salinidades. Este fato, também foi observado por Mcavoy \& Klug (2005) ao estudarem a ecofisiologia de $U$. intestinalis $\mathrm{L} .(=E$. intestinalis) em ambientes estuarinos. De fato, Lobban e Harrison (1997) afirmam que a presença de macroalgas, exclusivamente marinhas, em salinidades reduzidas faz com que a pressão de turgor cresça e as células, por conseguinte, se expandam desde que suas paredes sejam elásticas o suficiente para suportar tal tensão. A força das paredes celulares e a habilidade das células de manterem seu potencial osmótico interno determinam a resistência da espécie à baixa salinidade. Tal situação modifica por completo a resposta da alga quando colocada em situação de estresse, comprometendo as suas funções, como, por exemplo, sua produção. Por outro lado, cabe lembrar que Ulva flexuosa e Cladophora vagabunda foram encontradas na laguna hiperhalina de Araruama, local com salinidade desde marinha (35) até 55 e em lagunas com salinidades estuarinas, variando de 18 a 20 (Reis \& Yoneshigue-Valentin 1996; Pedrini 1980; Coutinho \& Seeliger 1984).

Quanto à resposta de Polysiphonia subtilissima na salinidade 15 , demonstrada pela sua maior produtividade, o controle da pressão de turgor ou a resistência foi constatado para este gênero. Reed $(1983,1984)$ observou que na alga estuarina Polysiphonia lanosa (L.) Tandy, o ajuste do seu potencial osmótico interno por osmólitos, era auxiliado por mudanças no volume celular. Quando comparadas as suas contrapartes marinhas, as paredes celulares dos espécimes estuarinos são mais finas e menos rígidas, permitindo assim que a célula inche ou esvazie de acordo com o gradiente osmótico. Reed (1983) também comenta que a necessidade de adaptação a múltiplos estresses em pequena escala de tempo leva a uma seleção de espécies mais resistentes ou até mesmo uma regulação parcial da pressão de turgor. Tais espécies são freqüentemente 
encontradas em estuários e em zonas entremarés como as que são tratadas neste trabalho.

Uma vez duas das três espécies estudadas apresentaram uma redução na produtividade liquida, como observado nas salinidades de 10 e 25, supomos que com a abertura do canal do Jardim de Alah poderão ocorrer modificações na dinâmica das populações de algas locais. Entretanto, existem exemplos de ampla resistência, como observado por Gessner \& Schramm (1971), com U. lactuca L., conhecida como espécie eurihalina, a qual quando foi submetida gradativamente da salinidade zero até a salinidade marinha, tem sua biomassa reduzida. Posteriormente, quando transferida para a água do mar, sua biomassa voltou aos valores normais.

Considerando que o alargamento do Canal do Jardim de Alah mudará o padrão atual da salinidade da Lagoa Rodrigo de Freitas, a dinâmica das populações de algas poderá sofrer mudanças. Isto pode ser exemplificado pelas duas espécies mais representativas de algas desta laguna, Cladophora vagabunda e Polysiphonia subtilissima, testadas neste estudo, que mostraram uma redução na produtividade liquida quando expostas a uma salinidade maior.

\section{Agradecimentos}

À Fundação Estadual de Engenharia do Meio Ambiente - FEEMA por conceder dados ambientais da laguna e, em especial, à $\mathrm{Dr}^{\mathrm{a}}$ Cristina Aparecida Gomes Nassar pelas inúmeras sugestões durante a realização desta pesquisa.

\section{REFERÊNCIAS BIBLIOGRÁFICAS}

COPPE. 2006. Periódicos COPPE, artigo 558. Acessado em 10 de janeiro de 2006. http:/ /www.planeta.coppe.ufrj.br/ artigo.php?artigo $=558$.

Coutinho, R. \& Seeliger U. 1984. Seasonal occurrence and growth of benthic algae in the Patos Lagoon estuary, Brazil. Estuarine. Coastal and Shelf Science 23(6): 889-900.

Czaplewski, L. R. \& Parker M., 1974. Use of a BOD Oxygen Probe for Estimating
Primary Productivity. Limnology and Oceanography 18(1): 152-154.

FEEMA. 2002. Qualidade da água da Lagoa Rodrigo de Freitas. Relatório de resultados obtidos através de campanhas de monitoramento e inspeções realizadas no período de Fevereiro/2000 a Maio/2002, 40p.

Gessner, F. \& Schramm, W. 1971. Salinity: plants. In: Kinne, O. (org.). Marine Ecology. Pp. 705-820.

Hayden, H. S.; Blomster, J.; Maggs, C. A.; Silva, P. C.; Stanhope, M. J. \& Waaland, J. R. 2003. Linneaus was right all along: Ulva and Enteromorpha are not distinct genera. European Journal of Phycology 38: 277-294.

Kim, K. Y. \& Lee, I. K. 1996. The germling growth of Enteromorpha intestinalis (Chlorophyta) in laboratory culture under different combinations of irradiance and salinity and temperature and salinity. Phycologia 35: 327-331.

Loureiro, R. R. 2007. Caracterização das assembléias de macroalgas da Lagoa Rodrigo de Freitas, Rio de Janeiro, Brasil. Dissertação de Mestrado. Instituto de Pesquisas Jardim Botânico do Rio de Janeiro, Rio de Janeiro, 67p.

Martins, I.; Oliveira, J. M.; Flindt, M. R. \& Marques, J. C. 1999. The effect of salinity on the growth rate of the macroalgae Enteromorpha intestinalis (Chlorophyta) in the Mondego estuary (west Portugal). Acta Oecologica 20: 259-265.

Mcavoy, M. K. \& Klug, J. L. 2005. Positive and negative effects of riverine input on the estuarine green alga Ulva intestinalis L. (= Enteromorpha intestinalis). Hydrobiologia 545: 1-9.

McGlathery, K. J.; Pedersen, M. F. \& Borum, J. 1996. Changes in intracellular nitrogen pools and feedback controls on nitrogen uptake in Chaetomorpha linum (Chlorophyta). Journal of Phycology 32: 393-401.

; Krause-Jensen, D.; Rysgaard, S. \& Christensen, P. B. 1997. Patterns of 
ammonium uptake within dense mats of the filamentous macroalga Chaetomorpha linum. Aquatic Botany 59: 99-115.

\& Pedersen, M. F. 1999. The effect of growth irradiance on the coupling of carbon and nitrogen metabolism in Chaetomorpha linum (Chlorophyta). Journal of Phycology 35: 721-731.

Oliveira, E. C.; Paula, E. J.; Plastino, E. M. \& Petti, R. 1995. Metodologias para o cultivo axênico de macroalgas marinas in vitro. In: Alveal, K; Ferrario, M. E.; Oliveira, E. C. \& Sar, E. (orgs.). Manual de métodos ficológicos. Universidad de Concepción, Chile. Pp. 429-455.

Oliveira, E. C.; Prirani, J. R. \& Giulietti A. M. 1983. The brazilian seagrasses. Aquatic Botany 16: 251-267.

Pedrini, A. G. 1980. Algas marinhas bentônicas da Baía de Sepetiba e arredores (Rio de Janeiro). Dissertação de Mestrado. Universidade Federal do Rio de Janeiro, Rio de Janeiro, 397p.

Poole, L. J. \& Raven, J. A. 1997. The biology of Enteromorpha. In: Round, F.E. \& Chapman D. J. (eds.). Progress in phycological research. Biopress Ltd., Bristol. 148p.

Ramus, J. \& Venable, M. 1987. Temporal ammonium patchiness and growth rate in Codium and Ulva (Ulvophyceae). Journal of Phycology 23: 518-523.

Reed, H. R.; Collins, J. C. \& Russel, G., 1980. The efects of salinity upon cellular volume of the marine alga Polysiphonia lanosa (L.) Tandy. Journal of Experimental
Botany 31: 1521-1531.

1983. The osmotic responses of Polysiphonia lanosa (L.) Tandy from marine and estuarine sites: Evidence for incomplete recovery turgor. Journal Experimental Marine Ecology 68: 169-193. .1984. The effects of extreme hyposaline stress upon Polysiphonia lanosa (L.) Tandy from marine and estuarine sites. Journal of Experimental Marine Ecology 76: 131-144.

Reis, R. P. \& Yoneshigue-Valentin, Y. 1996. Distribuição das macroalgas da Lagoa de Araruama, estado do Rio de Janeiro, Brasil. Revista Brasileira Botânica 19(1): 77-85.

Taylor, R.; Fletcher, R. L. \& Raven, J. A. 2001. Preliminary studies on the growth of selected 'green tide' algae in laboratory culture: effects of irradiance, temperature, salinity, and nutrients on growth rate. Botanica Marina 44: 327-336.

Wiencke, C.; Gorham, J.; Tomos, D. \& Davenport, J. 1992. Incomplete turgor adjustment in Cladophora rupestris under fluctuating salinity regimes. Estuarine, Coastal and Shelf Science. 34(4): 413-427.

\& Davenport, J. 1987. Respiration and photosynthesis in the intertidal alga Cladophora rupestris (L.) Kuetz. under fluctuating salinity regimes. Journal of Experimental Marine Biology and Ecology 114(2-3): 183-197. 